\title{
Sarcoidosis and smoking: An unlikely duo
}

\author{
Jonathan Kopel BS
}

\begin{abstract}
Sarcoidosis is a granulomatous pulmonary disorder in middle-age adults characterized by systemic proliferation of helper $T$ cells. Fewer than $10 \%$ of sarcoidosis patients have a family history, and many cases develop from interactions between environmental exposure and genetic polymorphisms. Several epidemiological studies suggest cigarette smoke with nicotine may prevent sarcoidosis. Given the many studies linking smoking to several diseases and long-term complications, recommending smoking or nicotine therapy for granulomatous lung diseases, such as sarcoidosis, remains a difficult proposition. More research is needed to isolate the chemical component(s) in cigarette smoke that might reduce the risk of sarcoidosis and determine efficacy and potential adverse effects. In addition, more investigation is needed to determine whether cigarette smoking actually lowers the risk of developing sarcoidosis.
\end{abstract}

Keywords: Sarcoidosis, smoking, causation, epidemiology

\section{INTRODUCTION}

Sarcoidosis is a granulomatous pulmonary disorder in middle-age adults characterized by systemic proliferation of helper $\mathrm{T}$ cells. ${ }^{1}$ Specifically, antigen activated macrophages and helper $T$ cells release inflammatory cytokines producing epithelioid granulomas, which can involve several organ systems, especially in the thorax. ${ }^{1}$ The diagnosis of sarcoidosis is confirmed with a biopsy showing epithelioid granulomas without caseating necrosis in a patient with a consistent clinical presentation. ${ }^{1}$ Sarcoidosis patients classically present with dry cough, dyspnea, and bilateral hilar lymphadenopathy and lung infiltrates on $\mathrm{x}$-ray imaging. ${ }^{1}$ Depending on the study method, the prevalence of sarcoidosis varies between 0.03-640 per 100,000 with a mortality rate of $1-6 \% .^{2}$ More than $30 \%$ of sarcoidosis patients may never have symptoms or

Corresponding author: Jonathan Kopel Contact Information: Jonathan.kopel@ttuhsc.edu DOI: 10.12746/swrccc.v8i34.679 will have spontaneous remissions; $10-30 \%$ have significant lung impairment. ${ }^{2}$ According to A Case-Control Etiologic Study of Sarcoidosis (ACCESS), female sarcoidosis patients had more ophthalmic, neurlogical, and dermatological complications, and male sarcoidosis patients had more hypercalcemia. ${ }^{3}$ Furthermore, the ACCESS found agricultural employment, automotive manufacturing, raising birds, pesticides, and microbial bioaerosols exposure were positively associated with the development of sarcoidosis. ${ }^{3}$ The major factors negatively associated with the development of sarcoidosis included a smoking history and pet ownership. ${ }^{2}$ Fewer than $10 \%$ of sarcoidosis patients have a family history; most cases result from interactions between environmental exposures and genetic polymorphisms in HLA antigens. ${ }^{1,4}$

Cigarette smoking is a major environmental risk factor for many pulmonary and systemic diseases, including, for example, rheumatoid arthritis, Crohn's disease, and multiple sclerosis. ${ }^{3,5,6}$ However, cigarette smoking has also shown to reduce the incidence and progression of ulcerative colitis. ${ }^{3,5,6}$ In recent years, several epidemiological studies have suggested that cigarette smoke with nicotine may prevent sarcoidosis. ${ }^{3,5,6}$ 
Table 1. Studies Examining the Association between Sarcoidosis and Smoking

\begin{tabular}{|l|l|l|c|c|c|c|}
\hline Author & Year & Country & $\begin{array}{c}\text { Number of } \\
\text { Patients }\end{array}$ & $\begin{array}{c}\text { Odds } \\
\text { Ratio }\end{array}$ & $\begin{array}{c}\text { 95\% Confidence } \\
\text { Interval }\end{array}$ & $\begin{array}{c}\text { Association } \\
\text { with Smoking }\end{array}$ \\
\hline Gupta $^{12}$ & 2010 & India & 196 & 0.75 & $0.35-1.56$ & No \\
\hline Ungprasert $^{7}$ & 2016 & USA & 332 & 0.34 & $0.23-0.50$ & Yes \\
\hline Newman $^{6}$ & 2004 & USA & 706 & 0.62 & $0.50-0.77$ & Yes \\
\hline
\end{tabular}

\section{SARCoIDOSIS AND SMOKING}

As shown in Table 1, an important study of 277,777 Swedish construction workers from 1978-1993 identified 342 patients with sarcoidosis using laboratory data and chest $\mathrm{x}$-rays. This study reported a negative association between smoking history and sarcoidosis. ${ }^{5}$ Furthermore, the study suggested that the non-nicotinic components of cigarette smoke are likely important in the relationship between smoking and sarcoidosis. Case control studies of smokers, most importantly ACCESS, have reported a decreased risk for developing sarcoidosis in smokers compared to non-smokers. ${ }^{3,7-11}$ The ACCESS was designed to test the hypotheses that environmental and occupational exposures are associated with sarcoidosis. ${ }^{3}$ The study included ten medical centers which recruited 706 newly diagnosed sarcoidosis patients with age-, race-, and sex-matched control subjects. ${ }^{3}$ The patients were subsequently questioned regarding occupational and nonoccupational exposures. ${ }^{3}$ The study discovered that smokers had a reduced odds (odds ratio 0.62; $95 \%$ confidence interval $0.50-0.77$ ) of developing sarcoidosis compared to non-smokers. ${ }^{3,7-11}$ A similar study conducted in Olmsted County, Minnesota, examined 332 patients with sarcoidosis also reported a reduced odds ratio of sarcoidosis comparing current smokers with never smokers adjusted for age and sex (odds ratio $0.34 ; 95 \%$ confidence interval, $0.23-0.50)^{7}$

A recent case-control study from India examining 100 newly diagnosed sarcoidosis patients reported that 12 had a smoking history while 30 of the 196 healthy control subjects reported a smoking history. ${ }^{12}$ The study showed no change in the clinical presentation or severity of disease in sarcoidosis patients with a history of smoking. ${ }^{12}$ Specifically, a logistical regression analysis comparing newly diagnosed sarcoidosis patients and healthy controls showed no correlation between sarcoidosis and smoking. Furthermore, the odds ratio between smoking and sarcoidosis was estimated at 0.75 (95\% confidence interval, 0.351.56). ${ }^{3,7,12}$ It is uncertain whether nicotine or other components of cigarette smoke alone or in combination change the clinical presentation of sarcoidosis. ${ }^{13}$

A clinical report examining nicotine treatment in active pulmonary sarcoidosis patients showed reduced lung inflammation and immune hyperactivity with nicotine. ${ }^{14}$ Specifically, nicotine treatment suppressed regulatory T-cell function and restored Toll-like receptor responsiveness to physiological levels. ${ }^{14} \mathrm{~A}$ similar response to nicotine treatment was found in patients with interstitial pneumonitis, which also occurs in the early stages of sarcoidosis. ${ }^{6,15-19}$ Specifically, in-vivo and in-vitro studies on interstitial pneumonitis showed a similar immunosuppressive response with nicotine as those seen in sarcoidosis patients. ${ }^{20}$ Patients with sarcoidosis who smoked had reduced bronchovesicular bundle thickening, which appears as branching lines with a peri-bronchovascular distribution on computed tomography scans. ${ }^{21}$ Previous studies in sarcoidosis patients have correlated the severity of bronchovesicular bundle thickening with the pathologic and symptomatic progression of the disease..$^{21}$ Although the exact mechanism is unclear, recent experiments suggest smoking suppresses T-lymphocyte and macrophage phagocytic activity, which might interfere with granuloma formation observed in patients with sarcoidosis. ${ }^{7,22,23}$

\section{Evaluation of Smoking and Sarcoidosis USING tHe BRADFoRd-HILL CRITERIA}

However, the negative correlation between sarcoidosis and smoking does not prove a causative 
link until further investigation. ${ }^{24-26}$ Causality is a multifaceted concept with specific guidelines analyzing statistical and epidemiological data to support causal inference. ${ }^{25,26}$ Many risk-related claims or correlations are often based on subjective judgments rather than an objective analysis of the study populations and models. ${ }^{27}$ In many cases the risk claims and associations were unsupported on further investigation and meta-analysis. ${ }^{27}$ In response, the scientific and medical community has sought strong and consistent criteria for determining biologically plausible causal claims. ${ }^{27}$ As the English statistician Sir Austin Bradford Hill explained, "Our observations reveal an association between two variables, perfectly clear-cut and beyond what we would care to attribute to the play of chance. [However] What aspects of that association should we especially consider before deciding that the most likely interpretation of it is causation? In what circumstances can [one] pass from [an] observed association to a verdict of causation."24-26 In pursuit of this question, Hill developed a set of nine criteria, known as the Bradford Hill Criteria, to determine whether a causative link exists between two variables. ${ }^{24-26}$ As shown in Table 2, the criteria include: strength, consistency, specificity, temporality, biological gradient, plausibility, coherence, experiment, and analogy. ${ }^{24,26}$ In each criteria, the negative association between sarcoidosis and smoking requires additional large, randomized clinical trials to satisfy the Bradford Hill Criteria.
The strength of the association between sarcoidosis and smoking is reported using the odds ratio, which represents the probability of an event occurring divided by the probability of the event not occurring. ${ }^{28}$ The odds ratio is applied in case-control studies to increase statistical power and reduce confounding in epidemiological studies. ${ }^{28}$ Although the odds ratio is a valid measure of association, it is not a substitute for other statistical measures and should only be applied to case-control studies and logistical regressions. ${ }^{28}$ The odds ratio reported in studies on sarcoidosis measured between sarcoidosis and smoking has a range of 0.32 to 0.75 , which represents a modest negative association. ${ }^{3,7,12}$ Furthermore, only two of the three studies showed a significant negative association between smoking and sarcoidosis. Therefore, the strength between smoking and sarcoidosis is weak. ${ }^{3,7,12}$

With respect to consistency and specificity, the association between sarcoidosis and smoking remains uncertain. Two studies conducted in the United States (U.S.) showed a negative association while the casecontrol study in India showed no association. ${ }^{3,7,12}$ The differences may result from differences in awareness, diagnostic methods, and demographics between the U.S. and India. ${ }^{3,7,12}$ With respect to temporality, there is weak evidence supporting the causal link of smoking and the subsequent decrease in sarcoidosis. ${ }^{3,7,12}$ One study showed that the odds ratio was no different

\section{Table 2. Bradford-Hill Criteria ${ }^{26}$}

\begin{tabular}{|l|l|}
\hline Criteria & Description \\
\hline Strength & Is there a large difference in outcome between the exposed group and control? \\
\hline Consistency & Have several researchers in different locations, circumstances, and times observed the same effect? \\
\hline Specificity & Are there specific persons or locations associated with the outcome? \\
\hline Temporality & Does the cause always precede the effect? \\
\hline Biological Gradient & Does the effect show a dose-response curve? \\
\hline Plausibility & Does the observed causation fit with our current understanding of biological phenomena? \\
\hline Coherence & $\begin{array}{l}\text { Does the observed causation conflict with our understanding of other biological phenomena or } \\
\text { the natural history of the disease? }\end{array}$ \\
\hline Experiment & Does the observed association allow for predictions in an outcome? \\
\hline Analogy & Is there an analogous exposure and outcome? \\
\hline
\end{tabular}


between former smokers and never smokers (odds ratio $0.68 ; 95 \% \mathrm{Cl}: 0.45-1.01) .{ }^{7}$ However, none of the studies investigated whether other confounding environmental variables which may impact the association between smoking and sarcoidosis. ${ }^{3,7,12}$ Large scale, prospective studies are needed to determine and eliminate potential confounding variables underlying any possible association. . $^{3,12}$ Despite non-nicotinic components showing a potential role in preventing sarcoidosis, no study has investigated whether increase amounts of non-nicotinic or nicotinic cigarette components from different sources, such as e-cigarettes, influence the association between sarcoidosis and smoking. ${ }^{5}$

Furthermore, the plausibility, coherence, and experiment aspects of the sarcoidosis case-studies recommending smoking or nicotine therapy for granulomatous lung diseases, such as sarcoidosis, remains a difficult proposition. ${ }^{13}$ Specifically, smoking does not benefit current sarcoidosis patients who may have a worse clinical outcome compared with nonsmokers with sarcoidosis. ${ }^{5}$ A study examining sarcoidosis patients who smoked had an increased prevalence of obstructive lung disease and decreased diffusion capacities compared to non-smokers. ${ }^{29}$ Furthermore, clinicians who manage patients with cigarette-related diseases may be reluctant to believe that tobacco could reduce the risk of granulomatous lung disease. ${ }^{13}$ Finally, only a few clinical studies have examined potential link between smoking and sarcoidosis, and information available is probably inadequate to reach a conclusion. ${ }^{29}$ No study has investigated whether administering nicotinic or non-nicotinic components of smoking in a randomized clinical trial prevents developing sarcoidosis, and a study like this will never happen. Further research is also needed to determine whether analogous exposures produce a similar association or reverses when smoking is discontinued. Overall, the association and causal link between smoking and sarcoidosis is weak and requires further investigation.

\section{Conclusion}

This focus of this article was evaluating the negative association between sarcoidosis and smoking.
However, there is a growing interest in the development of novel treatments for sarcoidosis and other hypersensitivity reactions. Despite the widespread use corticosteroid therapy, no targeted pharmacological treatments have been approved for sarcoidosis in the United States. ${ }^{30}$ Furthermore, no prospective study has determined the proper dose, duration, or tapering of corticosteroids in chronic sarcoidosis patients. ${ }^{30}$ More investigation is needed to determine whether cigarette smoking lowers the risk of developing sarcoidosis and could provide a novel approach to investigate new treatments for chronic sarcoidosis patients. At present, the epidemiological data suggesting cigarette smoke may prevent and reduce sarcoidosis symptoms remain unclear. With the numerous studies documenting the long-term effects of smoking, current smokers with sarcoidosis should be encouraged to discontinue smoking and other tobacco-related products.

Article citation: Kopel J. Sarcoidosis and smoking: an unlikely duo. The Southwest Respiratory and Critical Care Chronicles 2020;8(34):47-51

From: The School of Medicine, Texas Tech University Health Sciences Center, Lubbock, Texas

Submitted: $2 / 3 / 2020$

Accepted: 4/7/2020

Reviewer: Rishi Raj MD

Conflicts of interest: none

This work is licensed under a Creative Commons

Attribution-ShareAlike 4.0 International License.

\section{REFERENCES}

1. Bargagli E, Prasse A. Sarcoidosis: a review for the internist. Intern Emerg Med 2018;13(3):325-331.

2. Thomeer M, Demedts M, Wuyts W. Epidemiology of sarcoidosis. In. Sarcoidosis: European Respiratory Society; 2005:13-22.

3. Newman LS, Rose CS, Bresnitz EA, et al. A case control etiologic study of sarcoidosis. Am J Resp Crit Care Med 2004; 170(12):1324-1330.

4. Moller DR, Rybicki BA, Hamzeh NY, et al. Genetic, immunologic, and environmental basis of sarcoidosis. Annals Am Thoracic Soc 2017;14(Supplement_6):S429-S436. 
5. Carlens C, Hergens M-P, Grunewald J, et al. Smoking, use of moist snuff, and risk of chronic inflammatory diseases. Am J Resp Crit Care Med 2010;181(11):1217-1222.

6. Vassallo R, Ryu J. Tobacco smoke-related diffuse lung diseases. Seminars Resp Crit Care Med 2008;29(06): 643-650.

7. Ungprasert P, Crowson CS, Matteson EL. Smoking, obesity and risk of sarcoidosis: A population-based nested casecontrol study. Resp Med 2016;120:87-90.

8. Douglas JG, Middleton WG, Gaddie J, et al. Sarcoidosis: a disorder commoner in non-smokers? Thorax 1986; 41(10):787-791.

9. Hance AJ, Basset F, Saumon G, et al. Smoking and interstitial lung disease. Annals NY Acad Sci 1986;465(1 Tenth Interna):643-656.

10. Murin S, Bilello KS, Matthay R. Other smoking-affected pulmonary diseases. Clinics Chest Med 2000;21(1):121-137.

11. Valeyre D, Soler P, Clerici C, et al. Smoking and pulmonary sarcoidosis: effect of cigarette smoking on prevalence, clinical manifestations, alveolitis, and evolution of the disease. Thorax 1988;43(7):516-524.

12. Jindal SK, Singh A, Joshi K, et al. Is tobacco smoking protective for sarcoidosis? A case-control study from North India. Chest 2006;130(4):128S.

13. Maier LA. Is smoking beneficial for granulomatous lung diseases? Am J Resp Crit Care Med 2004;169(8):893-895.

14. Julian MW, Shao G, Schlesinger LS, et al. Nicotine treatment improves Toll-like receptor 2 and Toll-like receptor 9 responsiveness in active pulmonary sarcoidosis. Chest 2013; 143(2):461-470.

15. Margaritopoulos GA, Harari S, Caminati A, et al. Smokingrelated idiopathic interstitial pneumonia: A review. Respirology 2015;21(1):57-64.

16. Vassallo R, Ryu JH. Smoking-related interstitial lung diseases. Clinics Chest Med 2012;33(1):165-178.

17. Costabel U, Bross KJ, Guzman J, et al. Predictive value of bronchoalveolar T cell subsets for the course of pulmonary sarcoidosis. Annals NY Acad Sci 1986;465(1 Tenth Interna): $418-426$.
18. Crystal RG. Pulmonary sarcoidosis: A disease characterized and perpetuated by activated lung T-lymphocytes. Annals Internal Med 1981;94(1):73.

19. Mitchell DN, Scadding JG, Heard BE, et al. Sarcoidosis: histopathological definition and clinical diagnosis. J Clin Path 1977;30(5):395-408.

20. Schuyler M, Gott K, Cherne AMY. Is IL12 necessary in experimental hypersensitivity pneumonitis? International J Experimental Path 2002;83(2):87-98.

21. Gerke AK, van Beek E, Hunninghake GW. Smoking inhibits the frequency of bronchovascular bundle thickening in sarcoidosis. Acad Radiol 2011;18(7):885-891.

22. Moller W. Smoking impairs alveolar macrophage activation after inert dust exposure. Toxicology Letters 1996;88(1-3): 131-137.

23. Harf RA, Ethevenaux C, Gleize J, et al. Reduced prevalence of smokers in sarcoidosis. Annals NY Acad Sci 1986;465(1 Tenth Interna):625-631.

24. Hill AB. The environment and disease: association or causation? J Royal Soc Med 2015;108(1):32-37.

25. Fedak KM, Bernal A, Capshaw ZA, et al. Applying the Bradford Hill criteria in the 21st century: how data integration has changed causal inference in molecular epidemiology. Emerging Themes Epidemiology 2015;12(1).

26. Weyland P, Grant W, Howie-Esquivel J. Does sufficient evidence exist to support a causal association between vitamin D status and cardiovascular disease risk? An assessment using hill's criteria for causality. Nutrients 2014;6(9):3403-3430.

27. Cox LA. Modernizing the Bradford Hill criteria for assessing causal relationships in observational data. Critical Rev Toxicology 2018;48(8):682-712.

28. Grimes DA, Schulz KF. Making sense of odds and odds ratios. Obst Gyn 2008;111(2, Part 1):423-426.

29. Krell W, Bourbonnais JM, Kapoor R, et al. Effect of smoking and gender on pulmonary function and clinical features in sarcoidosis. Lung 2012;190(5):529-536.

30. Judson M, Barba, Beegle, et al. Current and emerging pharmacological treatments for sarcoidosis: a review. Drug Design, Development Therapy 2013:325. 